erkannt werden können, wenn ein erheblicher Stimmenverlust unweigerlich mit der Oppositionsrolle für eine Wahlperiode lang verbunden sein würde. Nach Abschaffung der FünfProzent-Hürde können sich Wahlverlierer aber immer noch durch Zusammenarbeit mit Kleinparteien und Gruppierungen die Mehrheit sichern und sich im Nachhinein als Sieger fühlen. Das verschafft denjenigen, die schon lange im politischen Prozess agieren und das politische Handwerkszeug kennen, Vorteile: Sie sind nämlich gefordert, Mehrheitskoalitionen zusammenzubringen und zusammenzuhalten, was sie praktisch über Jahre unersetzlich macht. Der häufig kritisierte Parteienfilz wird so nur noch gefördert. (Ober-)Bürgermeister, die gegen eine solche Mehrheitskoalition, deren Parteien sie bei der Wahl nicht unterstützt haben, ihre Leitungsfunktion in der Stadt wahrnehmen müssen, tun sich schwer; die Effizienz der Verwaltung nimmt Schaden. Gerade die Performanz der lokalen Autoritäten ist aber für die Vertrauensbildung sehr wichtig. ${ }^{126}$ Für die Bürger als Betrachter von außen wird die Zurechenbarkeit von Entscheidungen immer schwieriger. Ihnen mag es nicht mehr wichtig sein zu wählen, weil sich nicht durch Wahlen, sondern erst bei den unvermeidbaren „Koalitionsverhandlungen“, also im Nachhinein, die Machtverhältnisse klären.

126 Vgl. Christian Schnaudt, Politisches Vertrauen, in: Jan van Deth / Markus Tausendpfund, a.a.O. (Fn. 1), S. 297 - 328, S. 324.

\title{
Politische Generationen, demographischer Wandel und Wahlverhalten in der Bundesrepublik Deutschland: Schicksalsjahre des deutschen Parteiensystems?
}

\author{
Carmen Schmidt und Jan Knipperts
}

Die unmittelbaren Nachkriegsjahre waren in Deutschland noch relativ geburtenschwach. Ab Mitte der 1950er Jahre stieg die Geburtenrate dann stark an und erreichte zu Beginn der 1960er Jahre mit über 2,5 Kindern je Frau den höchsten Stand der Nachkriegszeit. Dieser als „Babyboom“ bezeichnete Anstieg endete mit dem so genannten „Pillenknick“ ab Mitte der 1960er Jahre. Seither nimmt die Zahl der Geburten im früheren Bundesgebiet kontinuierlich ab und liegt seit Anfang der 1970er Jahre unterhalb der Sterberate, was in den kommenden fünfzig Jahren zu einem Bevölkerungsrückgang von voraussichtlich 12 bis 17 Millionen führen wird. ${ }^{1}$ Neben einem Bevölkerungsschwund führt diese Entwicklung zu erheblichen Veränderungen in der Altersstruktur: Bereits heute sind 20 Prozent der Deutschen 65 Jahre oder älter, und schon in den kommenden beiden Jahrzehnten wird sich der Anteil älterer Menschen deutlich vergrößern. Weiter verstärkt wird dieser Effekt durch eine stetig steigende Lebenserwartung. ${ }^{2}$

1 Vgl. Hans Peter Friedrich, Die Demographiestrategie der Bundesregierung, in: Michael Hüther I Gerhard Naegele (Hrsg.), Demografiepolitik - Herausforderungen und Handlungsfelder, Wiesbaden 2013, S. 46 - 48, S. 46.

2 Vgl. Elke Drepper-Cramer, Der demographische Wandel in den Kommunen: Antizipatorische politische Entscheidungen angesichts struktureller Umbrüche, Uelvesbüll 2011, S. 25. 
Diese unter dem Begriff des demographischen Wandels zusammengefasste Bevölkerungsentwicklung hat tiefgreifende und nachhaltige Auswirkungen auf allen Ebenen und verändert die Organisation von Gesellschaft, Wirtschaft und Politik. Durch Verschiebung der Altersgruppen im Elektorat hin zu einem immer größeren Anteil älterer Wähler geht die Bedeutung jüngerer Altersgruppen für den Wahlerfolg der Parteien zurück. Hinzu kommen eine niedrigere Wahlbeteiligung und geringere Parteienidentifikation der jüngeren Wähler.

Die Frage, welche Folgen diese Veränderungen für die Parteien und das deutsche Parteiensystem haben werden, wurde bereits mehrfach thematisiert, wobei vor allem die unterschiedliche Partizipation nach Altersgruppen oder der älteren gegenüber den jüngeren Wählern untersucht wurde. ${ }^{3}$ Hier soll das Wahlverhalten der verschiedenen Generationengruppen in der (west)deutschen Gesellschaft und der Generationenwandel vor dem Hintergrund der demographischen Veränderungen in seinen Auswirkungen auf das politische System analysiert werden. Das Konzept der politischen Generationen erscheint hierfür besonders geeignet.

\section{Generation und Wahlverhalten}

\subsection{Das Generationenkonzept}

Die Generationenforschung geht auf Karl Mannheim zurück, der bereits im Jahre 1928 in seiner Abhandlung „Das Problem der Generationen“ die Bedeutung des Generationenwandels für den Werte- und Kulturwandel theoretisch fundierte. Er verglich die Generationenlage mit der Klassenlage, die beide im sozialen Raum verortet sind. Die Zugehörigkeit ist nicht freiwillig, sondern wird im Falle der Klasse durch die ökonomische Lage, im Falle der Generation durch das Geburtsjahr bestimmt. Beiden gemeinsam ist auch, dass Individuen eine ähnliche Perspektive auf die politischen und gesellschaftlichen Ereignisse ausbilden können: im Falle der Klasse auf der Basis der gleichen ökonomischen Lage, im Falle der Generation auf Basis des gemeinsamen historischen und kulturellen Kontextes. Ebenso wie die gemeinsame Klassenlage nicht zwangsläufig zu einem gemeinsamen Klassenbewusstsein führt, muss die Generationenlage nicht ein „Generationenbewusstsein “ hervorbringen. Förderlich für die Ausbildung eines kollektiven Bewusstseins bestimmter Generationen sind demgemäß entscheidende Kollektivereignisse, die „kristallisierend“ wirken. ${ }^{4}$

Mannheim wandte sich entschieden gegen eine „naturalistisch quantifizierbare“ Abfolge der Generationen. In welchen zeitlichen Abständen die Generationen aufeinander folgen, hängt von der „auslösenden Kraft des gesellschaftlich-geistigen Prozesses“5 ab, wobei dieser ökonomisch oder kulturell determiniert sein kann.

3 Vgl. etwa Bettina Westle, Demografischer Wandel und politische Teilhabe, in: Bernhard Frevel (Hrsg.), Herausforderung demografischer Wandel, Wiesbaden 2004, S. 163 - 179; Jürgen W. Falter, Demografischer Wandel und Wahlverhalten: Studie zum Wahlverhalten älterer Bürger und zu den Folgen des demografischen Wandels in Rheinland-Pfalz, Projektbericht des Instituts für Politikwissenschaft der Johannes Gutenberg-Universität Mainz, http://www.rlp.de/fileadmin/ staatskanzlei/rlp.de/downloads/landesregierung/Demographischer_Wandel_und_Wahlverhalten. pdf (Abruf am 19. Oktober 2013).

4 Karl Mannheim, Das Problem der Generationen, in: ders., Wissenssoziologie: Auswahl aus dem Werk, Neuwied 1964 (1928), S. 509 - 565, S. 552.

5 Ebenda. 
Die Bedeutung der Generationenforschung liegt nach Mannheim darin, gesellschaftlichen Wandel zu erklären. Das „Gegenwärtiger-Sein“ der Jugend bedeutet demzufolge, dass aktuelle Probleme infolge des „potentiell neuartigen Zuganges“ anders gedeutet und interpretiert werden und damit auch zu neuartigen Lösungskonzepten führen, während die ältere Generation bei ihrer früheren Orientierung verharrt. Die Dynamik des gesellschaftlichen Wandels liegt daher in der Abfolge von Generationen begründet. ${ }^{6}$

\subsection{Das Generationenkonzept in der Wahlforschung}

Obgleich Mannheims Aufsatz einen Schlüsseltext für die disziplinübergreifende Generationenforschung darstellte, fand der Generationenbegriff erst mit den Forschungen zum Wertewandel und grünen Parteien Eingang in die politische Soziologie, die bis dahin von strukturellen Ansätzen wie der Cleavage-Theorie geprägt war. So argumentierte Inglehart ${ }^{7}$, dass ab den 1970er Jahren insbesondere unter den Jüngeren und besser Gebildeten fundamentale Veränderungen der Wertepräferenzen von materialistischen zu postmaterialistischen Werten stattgefunden hätten, die sich auf das politische Verhalten des Einzelnen und das politische System insgesamt auswirkten. Die Voraussetzungen dieses Wandels lagen primär in der wirtschaftlichen Entwicklung und im Ausbau des Wohlfahrtsstaates, die zu ökonomischer und sozialer Sicherheit für die Masse der Bevölkerung führten.

Gemäß Inglehart ${ }^{8}$ und Helmut Fogt ${ }^{9}$ reflektieren die persönlichen Werte zu einem großen Teil die Bedingungen, die in Kindheit und Jugend erlebt wurden. Im Unterschied zur Lebenszyklusthese, die zunehmenden Konservativismus mit steigendem Lebensalter prognostiziert, wird angenommen, dass die fundamentalen Werteprioritäten über den Lebensverlauf beibehalten werden. ${ }^{10}$ Demzufolge findet kultureller Wandel in einer Gesellschaft nur über den intergenerationellen Wandel statt und wirkt sich nur allmählich und über eine längere Zeitspanne hinweg aus. Dies wurde auch als These von der generationellen Wasserscheide bezeichnet, derzufolge ab den 1970er Jahren ein fundamentaler Wandel der Werteeinstellungen stattgefunden habe, der sich immer weiter fortsetze und damit nicht auf eine Generation beschränkt sei. ${ }^{11}$

6 Vgl. ebenda, S. $539 \mathrm{ff}$.

7 Vgl. Ronald Inglehart, The Silent Revolution: Changing Values and Political Styles Among Western Publics, Princeton 1977.

8 Vgl. ders., Wertewandel in den westlichen Gesellschaften: Politische Konsequenzen von materialistischen und postmaterialistischen Prioritäten, in: Helmut Klages / Peter Kmieciak (Hrsg.), Wertewandel und gesellschaftlicher Wandel, Frankfurt am Main / New York 1979, S. 279 - 316, S. 280.

9 Vgl. Helmut Fogt, Politische Generationen. Theoretische Bedeutung und empirisches Modell, Opladen 1982, S. 21.

10 So ist insbesondere für die Mittelschicht festgestellt worden, dass mit zunehmendem Lebensalter häufig aufgrund eines gestiegenen Einkommens eine Erhöhung des sozialen Status einher geht, wodurch eine Tendenz zum Konservativismus erzeugt wird; in die gleiche Richtung wirkt auch das Vorhandensein von Kindern in Haushalten. Vgl. hierzu Seymour M. Lipset, Soziologie der Demokratie, ins Deutsche übertragen von Otto Kimminich, Neuwied / Berlin 1962, S. $295 \mathrm{ff}$.

11 Vgl. Markus Klein / Kai Arzheimer, Grau in Grau: Die Grünen und ihre Wähler nach eineinhalb Jahrzehnten, in: Kölner Zeitschrift für Soziologie und Sozialpsychologie, 49. Jg. (1997), H. 4, S. $650-673$, S. $652 \mathrm{f}$. 
Der von Inglehart u.a. prognostizierte Wertewandel sollte somit einerseits zu verstärkten Präferenzen der Wähler für eine „neue“ postmaterialistische Politik führen, andererseits zu einem graduellen Absinken von Parteiloyalitäten, da Jungwähler mit postmaterialistischen Einstellungen sich von den existierenden Parteien der „alten“, materiellen Politik nicht mehr angesprochen fühlen. Damit einher geht eine Ablehnung der herkömmlichen Formen politischer Partizipation, insbesondere der Beteiligung an Wahlen; stattdessen werden neue Formen der unkonventionellen Partizipation vorgezogen. ${ }^{12}$

Verschiedene Altersgruppen können somit Parteipräferenzen je nach den markanten Erfahrungen ihrer Generation entwickeln. Dies kann zu einem generationenspezifischem Wahlverhalten in dem Sinne führen, dass ältere Menschen die Ideen ihrer Jugend beibehalten, während jüngere Generationen andere Anschauungen vertreten. Somit können sich bestimmte Werteprioritäten von bestimmbaren Generationen durch die $\mathrm{Zu}$ - beziehungsweise Abnahme dieser Generationen an der wahlberechtigten Bevölkerung auf politische Systeme auswirken. ${ }^{13}$ Folglich erreichen die Auswirkungen ihren Höhepunkt, wenn eine bestimmte Generation die Mehrheit im Elektorat stellt, und lassen nach, wenn andere Generationen allmählich nachrücken. Auch Russell S. Dalton argumentiert, dass jede Generation unverwechselbare Werteprioritäten aufweist, da sich die akkumulierten Erfahrungen von Generation zu Generation unterscheiden. ${ }^{14}$ Die großen Unterschiede, die die Erfahrungen der Generationen kennzeichnen, führen demzufolge auch zu generationenspezifischen Parteiprioritäten.

\section{Generationengruppen in der Bundesrepublik Deutschland}

\subsection{Generationengruppen und gesellschaftlich-ökonomische Rahmenbedingungen}

Derzeit können unter der wahlberechtigten Bevölkerung in der Bundesrepublik vier wesentliche Generationengruppen unterschieden werden: (1) die direkte Nachkriegsgeneration, geboren ab Mitte der 1930er bis Anfang der 1950er Jahre; (2) die Wohlstandsgeneration, geboren Anfang der 1950er bis Mitte der 1960er Jahre; (3) die zwischen Mitte der 1960er bis Anfang der 1980er Jahre geborene Generation X, benannt nach dem gleichnamigen Episodenroman von Douglas Coupland aus dem Jahr 1991, der die Wertekultur der damaligen Jugendgeneration in Abgrenzung gegen die vorherige Wohlstandsgeneration beschreibt ${ }^{15}$ und (4) die sich anschließende Generation Y, wie die nach 1981 Geborenen in der Fachzeitschrift „Ad Age“ aus dem Jahr 1993 bezeichnet wurden. ${ }^{16}$

12 Vgl. Kai Hildebrandt / Russell S. Dalton, Die neue Politik: Politischer Wandel oder Schönwetterpolitik, in: PVS, 17. Jg. (1977), H. 2/3, S. 230 - 256.

13 Vgl. zum Beispiel Ronald Inglehart, a.a.O. (Fn. 7).

14 Vgl. Russell S. Dalton, Wertewandel oder Wertewende: Die Neue Politik der Parteienpolarisierung, in: Max Kaase (Hrsg.), Wahlen und politisches System: Analysen aus Anlaß der Bundestagswahl 1980, Opladen 1983, S. 427 - 454, S. 450.

15 Der Roman mit dem Titel „Generation X: Geschichten für eine immer schneller werdende Kultur“ handelt von drei Universitätsabsolventen, die ihren Lebensunterhalt mit schlecht bezahlten Jobs bestreiten, für die sie völlig überqualifiziert sind und in ihrer Selbstwahrnehmung eine Art „ArmutJet-Set" darstellen.

16 Die darauf folgende Generation Z gehört heute noch nicht zur wahlberechtigten Bevölkerung. 
Die genauen Generationenkohorten lassen sich indes nicht auf ein Jahr genau angeben und variieren je nach Autor ${ }^{17}$, was auch darin begründet liegt, dass ökonomische und soziale Prozesse je nach Wohnort (Stadt vs. Land) schneller oder langsamer ablaufen und gleichermaßen prägende Erfahrungen auch innerhalb eines Landes zeitversetzt eintreten können. So haben auch Martin Klaffke und Andreas Parment argumentiert, dass sich Werte und Einstellungen nicht ab einem gewissen Geburtsdatum abrupt ändern und sich Personen einer früheren Generation durchaus wie die nachfolgende verhalten können und umgekehrt. ${ }^{18}$ Es gibt daher auch beim Generationenansatz gewisse Grauzonen. Dessen ungeachtet bietet das auf Aggregatdaten beruhende Generationenkonzept, gerade wegen der hiermit verbundenen Komplexitätsreduktion, ein wichtiges Instrument, um Tendenzen und Entwicklungen in einer Gesellschaft besser zu verstehen. ${ }^{19}$

Die hier gewählte Geburteneinteilung der Generationen, die in Tabelle 1 wiedergegeben ist, orientiert sich an den gängigen Modellen sowie dem Datum 1965, dem Zeitpunkt des so genannten „Pillenknicks“, ab dem die Geburtenrate plötzlich abfiel und einen umfassenden Prozess des demographischen Wandels einleitete. ${ }^{20}$

Die Nachkriegsgeneration, zunächst geprägt von Kriegszerstörung und materieller Unsicherheit, erlebte den Wiederaufbau Deutschlands, die Demokratisierung und steigenden Wohlstand. Innerhalb des Parteiensystems war die Gründungs- und Konsolidierungsphase zu Anfang der 1960er Jahre abgeschlossen, wobei sich die zwei großen Volksparteien im

17 Manchmal werden Nachkriegs- und Wohlstandsgeneration angesichts der Tatsache, dass es sich um geburtenstarke Jahrgänge handelt, als Babyboomer-Generation zusammengefasst (geboren zwischen 1945 und 1964) und von den Traditionalisten (geboren zwischen 1928 und 1945) unterschieden (so Lynda Gratton, Job Future - Future Jobs. Wie wir von der neuen Arbeitswelt profitieren, München 2012; ähnlich auch Danielle Sacks, Scenes from the Culture Clash, in: Fast Company, Januar/Februar 2006, H. 102, S. 72 - 77, http://www.fastcompany.com/54444/scenesculture-clash (Abruf am 10. Mai 2013)). Dem widerspricht Roland Kecskes, der argumentiert, dass die Babyboomer, die eine Phase des stetigen Wohlstands und des Aufstiegs Deutschlands zu einer der führenden Industrienationen der Welt erlebten, eher zwischen 1952 und 1966 geboren wurden. Er plädiert daher auch für die Bezeichnung „Wohlstandsgeneration“ (ders., Auf der Suche nach einem kohärenten Qualitätsversprechen: Die junge, flexible Generation zwischen öffentlicher Inszenierung und privater Authentizitätssuche, in: GFK SE Consumer Panel, Juli 2012, S. 5 - 23, S. 6 ff.). Die Generation X wird als zwischen 1961 und 1965 geboren verortet (Stephen Earl Bennett / Stephen C. Craig, Generations and Change: Some Initial Observations, in: dies. (Hrsg.), After the Boom: The Politics of Generation X, Lanham 1997, S. 1 - 19, S. 18). Nur Michael D. Martinez setzt hier einen deutlich späteren Zeitraum an und definiert die Generation X als die nach 1972 geborenen (ders., Losing Canada? Generation X and the Constitutional Crisis, in: ebenda, S. 145 - 166). Auch für die Generation Y gibt es keine allgemeingültige zeitliche Einordnung. Schlug die Zeitschrift „Ad Age“ die Alterkohorten ab 1981 vor, plädiert zum Beispiel Sacks für die Geburtenjahrgänge 1978 bis 2000.

18 Vgl. Martin Klaffe / Andreas Parment, Herausforderungen und Handlungsansätze für das Personalmanagement von Millenials, in: Martin Klaffke (Hrsg.), Personalmanagement von Millenials: Konzepte, Instrumente und Best-Practice-Ansätze, Wiesbaden 2011, S. 3 - 21, S. 7.

19 Hier muss wie bei allen Analysen auf der Aggregatdatenebene berücksichtigt werden, dass von Merkmalen der Kollektivebene (der Generationen) nicht zwangsläufig auf Merkmale der Individualebene (der Wähler) geschlossen werden kann. Zur Problematik des „ökologischen Fehlschlusses" vgl. William S. Robinson, Correlations and the Behavior of Individuals, in: American Sociological Review, 15. Jg. (1950), H. 3, S. $351-357$.

20 Vgl. Bundesministerium für Arbeit und Soziales, Glossar: Demografisch markante Perioden, http://www.der-demograf.de/index.php?id=84\&area=af (Abruf am 13. Mai 2013). 


\begin{tabular}{|c|c|c|}
\hline \multicolumn{3}{|c|}{ Tabelle 1: Übersicht über die Generationengruppen der Untersuchung } \\
\hline Kohorte & Bezeichnung & Eintritt in das Elektorat \\
\hline 1934 bis 1949 & Nachkriegsgeneration & 1954 bis 1969 \\
1950 bis 1964 & Wohlstandsgeneration & 1970 bis 1982 \\
1965 bis 1980 & Generation X & 1983 bis 1998 \\
1981 bis 1995 & Generation Y & 1999 bis 2013 \\
\hline Anmerkung: Das Wahlalter betrug bis zur Wahl 1972 21 Jahre. \\
Quelle: Eigene Zusammenstellung.
\end{tabular}

Bundestag und in den Landtagen etablieren konnten, die CDU/CSU, der die Wirtschaftskompetenz zugeschrieben wurde, und die SPD, die sich für den Ausbau des Sozialstaates und die ökonomische Teilhabe der Arbeitnehmer einsetzte. Die einzige verbliebene Kleinpartei FDP wurde zum begehrten Koalitionspartner und damit zum „Zünglein an der Waage" 21 .

Für die Wohlstandsgeneration setzte sich diese Phase des stetigen Wohlstandes, der Stabilität des „Zweieinhalb-Parteiensystems“ und des Aufstiegs Deutschlands zu einer der führenden Industrienationen der Welt fort. Angesichts des raschen Wirtschaftswachstums waren die Aufstiegschancen nahezu unbegrenzt. Die Mitglieder dieser Generationen profitierten von einer starken Nachfrage nach Arbeitskräften, dem Ausbau des Bildungssystems und des Wohlfahrtsstaates. Auf der Werteebene weisen beide Generationen daher eine hohe Leistungsorientierung, einen starken Berufsbezug sowie die Suche nach Beständigkeit auf. Angesichts der Tatsache, dass die Wirtschaftswunderjahre einen weitgehenden Ausbau des Sozialstaats möglich machten und in der Gesellschaft weniger ökonomische Verteilungskämpfe, sondern eher ein harmonisches Miteinander im Vordergrund stand, waren diese Generationen stark durch kollektivistische Werte geprägt. ${ }^{22}$

Die Generation X erfuhr wachsende Individualisierung, aber auch ein erhöhtes Risiko zu scheitern, was sich in steigenden Arbeitslosenzahlen in dieser Zeit manifestiert. Mit der ersten Ölkrise 1973 endete die Hochwachstumsphase. Für diejenigen, die in dieser Zeit sozialisiert wurden, ist eine mangelnde Bereitschaft zu dauerhaften Organisationsbindungen zu konstatieren. Daneben lässt sich eine erheblich nachlassende Bindungskraft der Kirchen feststellen. Für diese Veränderungen wird, neben der Abschwächung der traditionellen gesellschaftlichen Konfliktstrukturen, die zunehmende Individualisierung und Pluralisierung verantwortlich gemacht. Insbesondere die „Herauslösung“ aus historisch vorgegebenen Sozialformen und -bindungen im Sinne traditionaler Herrschafts- und Versorgungszusammenhänge sowie der Verlust von traditionellen Sicherheiten kennzeichnen die in dieser Zeit geprägte Generation X.23

In der Struktur der Erwerbstätigkeit ist während ihrer Sozialisationsphase eine deutliche Verschiebung vom primären und sekundären hin zum tertiären Wirtschaftssektor sowie eine starke Flexibilisierung des Arbeitsmarktes bei stetig sinkenden Arbeitsmarktchancen auszu-

21 Olaf Jandura, Kleinparteien in der Mediendemokratie, Wiesbaden 2007, S. 28.

22 Vgl. Martin Klaffke / Andreas Parment, a.a.O. (Fn. 18), S. 6 ff.

23 Vgl. Ulrich von Alemann / Tim Spier, Deutsche Parteien unter veränderten Rahmenbedingungen, in: Uwe Andersen (Hrsg.), Parteien in Deutschland. Krise oder Wandel?, Schwalbach 2009, S. 39 -61, S. 43. 
machen. ${ }^{24}$ Im Vordergrund steht für diese Generation die Spannung zwischen Wahlmöglichkeit und Scheitern, weshalb sie auch als „verunsicherte Generation“ bezeichnet wurde. ${ }^{25}$ Die politische Sozialisation erfolgte in Zeiten der neuen sozialen Bewegungen und der Gründung der Grünen als neuer Alternative im Parteiensystem, die explizit postmaterialistische Werte vertrat.

Die Generation Y ist von einer hohen Flexibilität auf dem Arbeitsmarkt geprägt und hat nur geringe Hoffnung auf eine, für frühere Generationen selbstverständliche, kontinuierliche Erwerbsbiographie; vielmehr muss sie mit ständigen Diskontinuitäten und Brüchen rechnen, weshalb sie auch als „flexible Generation“ bezeichnet wird. ${ }^{26}$ Ihre Angehörigen erlebten in ihrer Jugend die deutsche Wiedervereinigung und den Wegfall des ideologisch aufgeheizten Ost-West-Konfliktes. Daneben ist die wirtschaftliche, politische und kulturelle Globalisierung und die damit einhergehende technologische Entwicklung ein weiteres prägendes Moment für diese Generation: So haben neue und immer leistungsstärkere Möglichkeiten in der Kommunikationstechnologie, wie Internet und Mobilfunk, die gesellschaftlichen Kommunikationsmuster grundlegend verändert. Diese Veränderungen laufen deutlich schneller ab, als das institutionelle System zu entsprechendem Wandel imstande ist. Das System folgt somit noch einer älteren Logik, während die Individuallagen durch eine hohe Pluralisierung von Rollen gekennzeichnet sind, die nur noch zum Teil den Erwartungen der Institutionen entsprechen. ${ }^{27}$ Innerhalb des Parteiensystems ist seit der deutschen Wiedervereinigung vor allem durch die Etablierung der Linkspartei eine Zunahme der Fragmentierung zu konstatieren. Daneben entstanden neue Gruppierungen wie die Piratenpartei, die mit Themen wie der Freiheit des Internets vor allem auf junge Wähler zielt. ${ }^{28}$ Auch ist argumentiert worden, dass die Globalisierung zu neuen strukturellen Konflikten führt, nämlich zwischen den Gewinnern der Globalisierung und einer heterogenen Klasse von Verlierern, die ein Wählerpotential für neue populistische Parteien stellt. ${ }^{29}$

\subsection{Politische Generationen in der Bundesrepublik: vier Thesen}

Wie ausgeführt müssen die Mitglieder einer Generation nicht zwangsläufig auch ein gemeinsames politisches Bewusstsein entwickeln. Sie können erst dann als politische Generationen

24 Vgl. Viola Neu, Bundestagswahl in Deutschland am 27. September 2009. Wahlanalyse, Berlin 2009, S. 16.

25 Vgl. Roland Kecskes, a.a.O. (Fn. 17).

26 Vgl. ebenda.

27 Vgl. Heiko Biehl, Parteienmitglieder im Wandel. Partizipation und Repräsentation, Wiesbaden 2005, S. 19.

28 Nach der Bundestagswahl 2009, bei der sie einen überraschenden Stimmenanteil von zwei Prozent erzielte, konnte sich die Partei in der Folgezeit in verschiedenen Landtagen etablieren. Überraschend war allerdings der anschließende Absturz in der Gunst der Wählermeinung, die sich im Wahldebakel bei der Wahl zum Niedersächsischen Landtag am 20. Januar 2013 manifestierte.

29 Vgl. Hanspeter Kriesi, Movements of the Left, Movements of the Right: Putting the Mobilization of Two New Types of Social Movements into Political Context, in: Herbert Kitschelt / Peter Lange I Gary Marks / John D. Stephens (Hrsg.), Continuity and Change in Contemporary Capitalism, Cambridge 1999, S. 398 - 423, S. 403 f.; Hanspeter Kriesi / Edgar Grande / Martin Dolezal / Marc Helbling / Dominic Höglinger / Swen Hutter / Bruno Wüest (Hrsg.), Political Conflict in Western Europe, Cambridge 2012. 
bezeichnet werden, wenn sich generationenspezifisches Wahlverhalten feststellen lässt. Unter Berücksichtigung der für die einzelnen Generationen spezifischen sozio-ökonomischen Rahmenbedingungen und den vorliegenden Untersuchungen zum Wahlverhalten lassen sich folgende Thesen zur politischen Partizipation der Generationen formulieren, die vorliegende Analyse leiten:

(1) Die direkte Nachkriegsgeneration (geboren bis Anfang der 1950er Jahre) ist geprägt von materieller Unsicherheit bei sich gleichzeitig stetig verbessernden ökonomischen Rahmenbedingungen. Da für sie der wirtschaftliche Aufstieg und die materielle Absicherung im Vordergrund gestanden haben dürften, sollte eine Tendenz zur CDU/ CSU bestehen, die als Partei für den Aufbau der Wirtschaft stand.

(2) Die Wohlstandsgeneration (geboren Anfang der 1950er bis Mitte der 1960er Jahre), ist geprägt ist von wirtschaftlichem Aufstieg und sozialer Sicherheit. Diese sollte wie ihre Vorgängergeneration eine materialistische Werteeinstellung aufweisen. Da für sie nicht mehr der Wiederaufbau der Wirtschaft, sondern eher die ökonomische Teilhabe im Vordergrund stand, sollte diese Generation tendenziell eine Affinität zur SPD aufweisen, die gemeinsam mit den Gewerkschaften für Lohnerhöhungen und mehr soziale Wohlfahrt eintrat. Die Mehrheit der Mitglieder dieser beiden genannten Generationengruppen zählt zu den geburtenstarken Babyboomer-Jahrgängen.

(3) Die darauf folgende Generation X (geboren ab Mitte der 1960er Jahre), die angesichts des „Pillenknicks“ zur Mitte der 1960er Jahre deutlich geburtenschwächer ist und deren Sozialisation unter deutlich verschlechterten Arbeitsmarktbedingungen bei gleichzeitig steigendem Bildungsniveau erfolgte, sollte im Vergleich zu den Vorgängergenerationen eine fundamental unterschiedliche, postmaterielle Werteeinstellung und daher deutlich geringere Bindungen an die Parteien der „alten Politik“ aufweisen ebenso wie eine nachlassende Neigung, sich an Wahlen als konventioneller Form der Partizipation zu beteiligen. Angesichts ihrer postmateriellen Einstellungen sollte diese Generation eine Tendenz zur Wahl der Grünen zeigen.

(4) Die Generation Y (geboren ab den 1980er Jahren), wurde nach dem Ende des ideologisch geprägten kalten Krieges unter den Bedingungen der Globalisierung sozialisiert. Gemäß der Theorie der generationellen Wasserscheide sollte auch diese Generation eine postmaterielle Werteeinstellung aufweisen und daher gegenüber den Parteien der „alten“ materiellen Politik eine eher ablehnende Haltung einnehmen, ebenso wie gegenüber konventionellen Formen der politischen Partizipation. Inwiefern sich der mit der Globalisierung einhergehende strukturelle Konflikt zwischen ihren Gewinnern und Verlieren auf das Wahlverhalten dieser Generation auswirkt, muss offen bleiben.

\section{Generationengruppen und politische Partizipation}

\subsection{Generelle Trends der quantitativen Verteilung der Generationengruppen}

Generationelles Wahlverhalten wirkt sich auf das Wahlergebnis vor allem dann aus, wenn eine bestimmte Generation zahlenmäßig einen hohen Anteil im Elektorat stellt. Daher sollen zunächst die generellen Trends der quantitativen Verteilung der Wahlberechtigten analysiert werden.

Abbildung 1 zeigt den Anteil der 18- bis 25-Jährigen im Vergleich zu den über 65-Jährigen an der wahlberechtigten Gesamtbevölkerung von 1970 bis zum Jahr 2040. Die zahlen- 


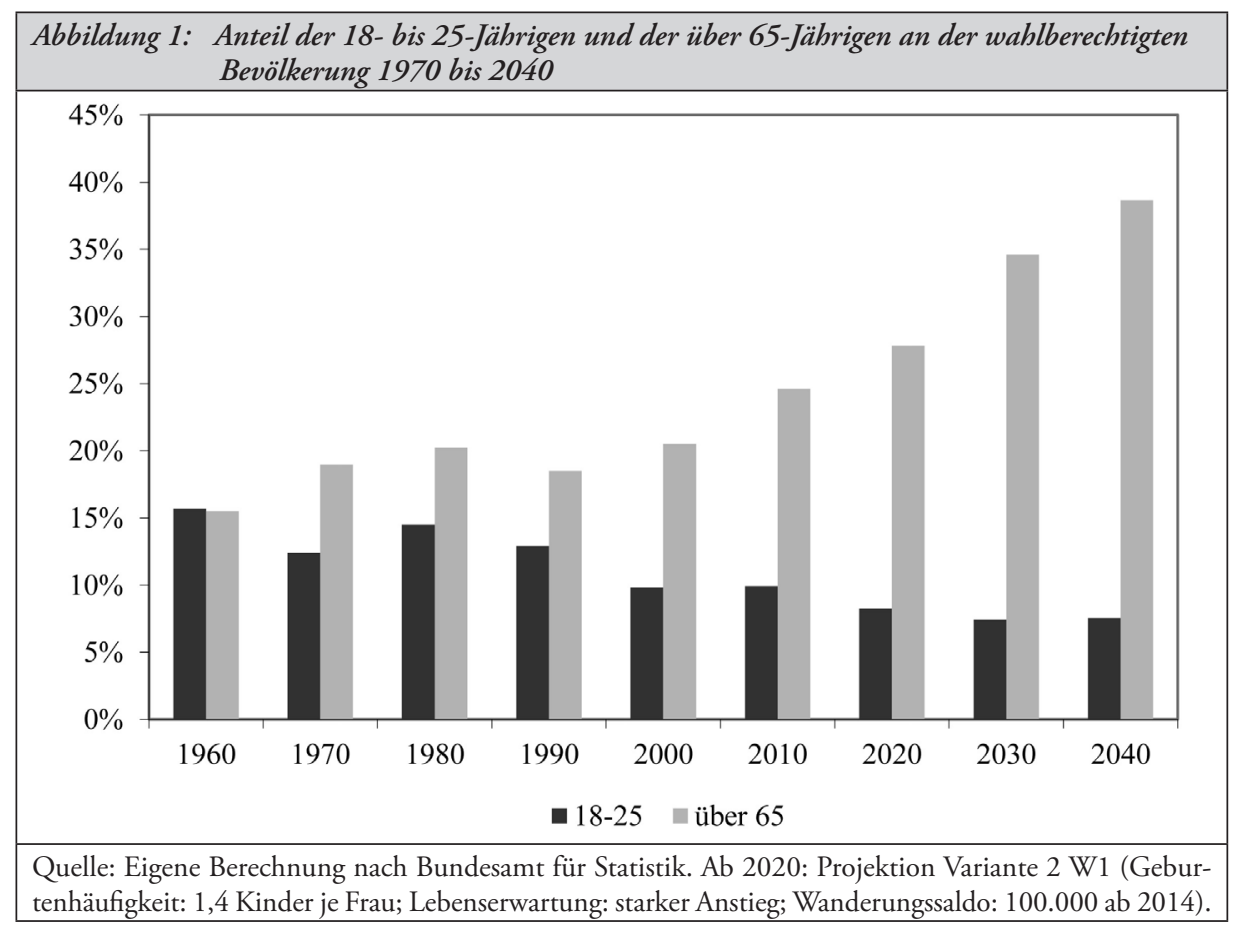

mäßig starke Wohlstandsgeneration wurde zwischen 1970 und 1982 wahlberechtigt. Alle Folgegenerationen machen angesichts des demographischen Wandels einen immer kleineren Anteil an den Wahlberechtigten aus, weshalb die Parteipräferenzen der jüngeren Generationen in immer geringerem Maße den Ausgang von Wahlen beeinflussen. Als die Wohlstandsgeneration 1970 in das Elektorat eintrat, hatte das Verhältnis der über 65-Jährigen zu dieser Generation noch 1,5:1 betragen. Im Jahr 2020 hingegen wird das Verhältnis dieser Altersgruppe zur jüngsten Kohorte bei 3,5:1 liegen. Man kann daraus folgern, dass die Wohlstandsgeneration in größerem Maße das politische System beeinflussen konnte, als dies einer der Nachfolgegenerationen möglich war. Zudem kann in Anlehnung an die Annahmen von Karl Mannheim geschlossen werden, dass sich durch den immer geringer werdenden Anteil der Jüngeren an der Bevölkerung generell die Dynamik des gesellschaftlichen Wandels in den letzten zwei Jahrzehnten deutlich verringert hat und noch weiter abnehmen wird.

Die Verteilung der hier zugrunde gelegten Generationengruppen am Elektorat ist in Abbildung 2 wiedergegeben. Zusammengenommen stellten Nachkriegs- und Wohlstandsgeneration (inklusiv früherer, noch im Elektorat vertretener Generationen) ${ }^{30}$ noch bis zum Jahr 2010 die Mehrheit der Wahlberechtigten, erst danach sinkt der Anteil unter 50 Prozent ab. Der Generationenwandel wird verdeutlicht, wenn man die Verteilung über die Zeit betrachtet. 1990 hatten diese Generationen noch 87 Prozent der Wahlberechtigten gestellt, im Jahr 2000 waren es noch 71 Prozent, 201054 Prozent und im Jahr 2020 werden es nur noch 45 Prozent sein. Nimmt man an, dass sich diese Generationen im Hinblick auf die

30 Inklusive früherer Generationen, die hier nicht betrachtet wurden. 


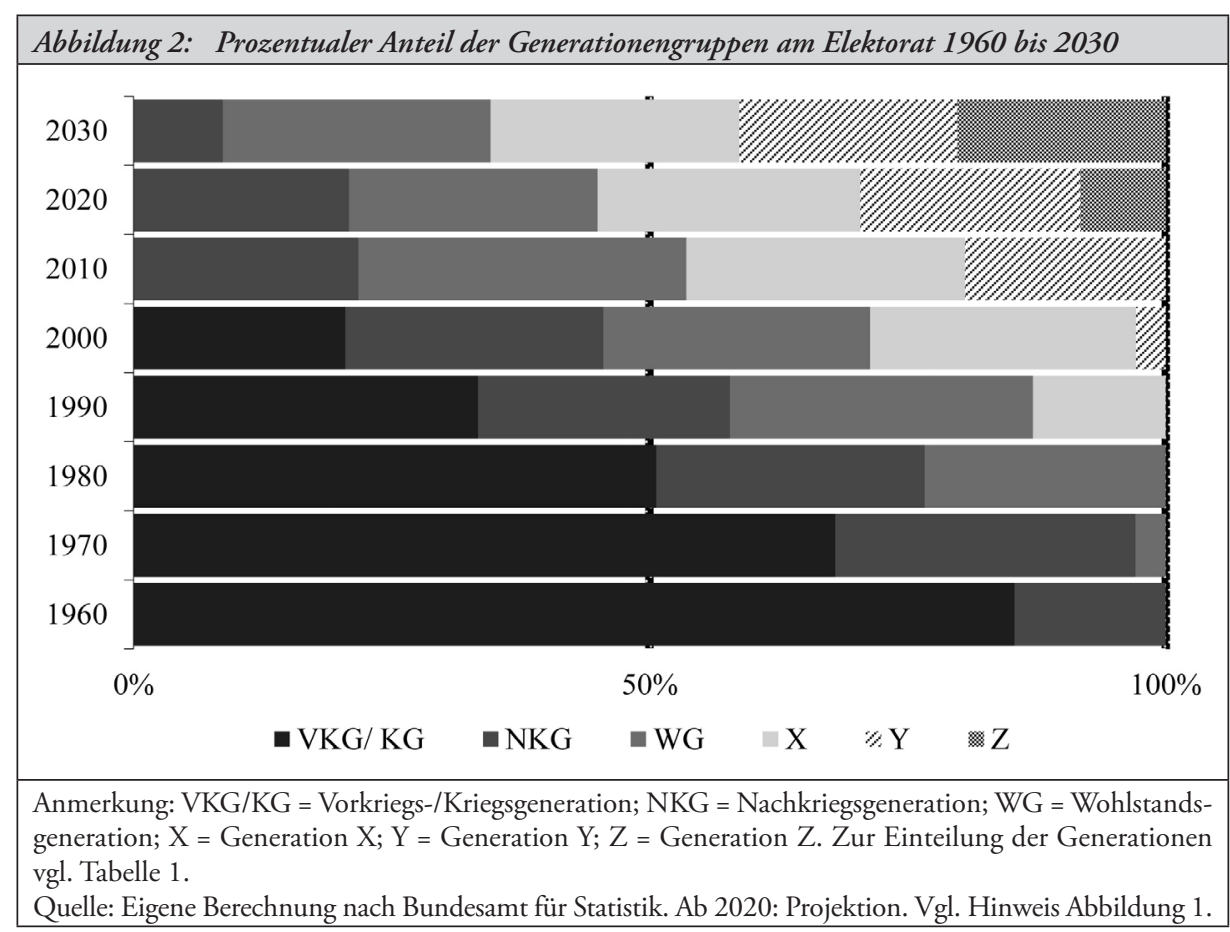

Parteienbindungen fundamental von den Folgegenerationen X und $\mathrm{Y}$ unterscheiden, so sind die Jahre zwischen 2010 und 2020 eine Wendezeit mit möglicherweise beträchtlichen Folgen für das deutsche Parteiensystem. Kurzfristig stellt sich der demographische Wandel somit als Generationenwandel dar, nämlich von den materialistisch geprägten Generationen des wirtschaftlichen Aufstiegs und gesellschaftlichen Wohlstands, die angesichts ihrer großen Zahl über eine lange Zeitspanne hinweg das politische System der Bundesrepublik dominieren konnten, hin zu den geburtenschwachen, von sich verschlechternden Arbeitsmarktbedingungen und den Prozessen der Globalisierung geprägten Generationen X und Y. Dieser Generationenwechsel ist für das politische System dann von Bedeutung, wenn sich für die Generationen ein je spezifisches Wahlverhalten feststellen lässt, was im folgenden Kapitel geklärt werden soll.

\subsection{Die politische Partizipation der Generationen im Spiegel der repräsentativen} Wahlstatistik

Die folgende Analyse des Wahlverhaltens der verschiedenen Generationengruppen in der Bundesrepublik Deutschland stützt sich auf die Ergebnisse der repräsentativen Wahlstatistik, die die allgemeine Wahlstatistik um Angaben getrennt nach Geschlechtern und Altersgruppen ergänzt. ${ }^{31} \mathrm{Da}$ die theoretischen Annahmen zu den politischen Generationen nur für das

$31 \$ 51$ Abs. 2 BWahlG. Sie wird vom Statistischen Bundesamt beziehungsweise den Statistischen Landesämtern durchgeführt. Aus circa 80.000 Wahlbezirken (ohne Briefwahlbezirke) wird durch 
alte Bundesgebiet gültig und angesichts unterschiedlicher historischer Erfahrungen nicht auf die neuen Bundesländer übertragbar sind, wurden im Folgenden nur die Ergebnisse für das frühere Bundesgebiet zugrunde gelegt.

Bei der Interpretation ist zu beachten, dass politische Partizipation aus dem Zusammenwirken verschiedener Faktoren resultiert. Es sollte daher unterschieden werden zwischen Langzeittrends, die durch das Wahlverhalten einer politischen Generation hervorgerufen werden, und Kurzzeit-Fluktuationen, die durch politisch-konjunkturelle Begleiterscheinungen einer bestimmten Wahl ausgelöst werden, wie beispielsweise die Wiedervereinigung zu Anfang der 1990er Jahre. 32

Im Hinblick auf das Wahlverhalten der Altersgruppe 21 bis 30 (Wahlen 1953 bis 1969) beziehungsweise der Altersgruppe 18 bis 25 (Wahlen 1972 bis 2009) ${ }^{33}$ lassen sich folgende Tendenzen feststellen:

(1) Bis 1965 ist unter den Jungwählern der Anteil für die CDU/CSU am höchsten und liegt zwischen 45 und 50 Prozent.

(2) Ab 1969 steigt der Anteil für die SPD deutlich und bleibt bis 1980 sehr hoch bei etwa 50 Prozent. Parallel dazu nimmt der Anteil für die CDU/CSU ab.

(3) Ab 1983 sinkt der Anteil der SPD stark, der Anteil für die Grünen hingegen steigt stark an. Auch der Anteil der CDU/CSU nimmt tendenziell weiter ab. Vermutlich aufgrund der deutschen Wiedervereinigung sinkt der Anteil der Grünen bei der Wahl im Jahr 1990 kurzfristig, wächst aber im Verlaufe der Zeit wieder an. Hier dürfte es sich somit um einen kurzfristigen Periodeneffekt gehandelt haben.

(4) Bei der Wahl 2009 zeigt sich eine dramatische Abnahme für die Großparteien SPD und CDU; alle kleineren Parteien hingegen schneiden überdurchschnittlich gut ab. Auch der Anteil für sonstige Parteien liegt mit 14,4 Prozent sehr hoch (vgl. Tabelle 2).

Diese Ergebnisse sind relativ konsistent mit den eingangs aufgestellten Thesen zum Wahlverhalten der Generationengruppen. Die Wohlstandsgeneration, die etwa um das Jahr 1970 in das Elektorat eintrat, zeigte eine eindeutige Präferenz für die SPD, während ihre Vorgängergeneration tendenziell die CDU/ CSU bevorzugte. Die Generation X, die ab Anfang der 1980er Jahre wahlberechtigt wurde, präferierte verstärkt die Grünen, deren Wähleranteile stetig zunahmen. Allerdings sollte nicht übersehen werden, dass der Partei, die von etwa 15 Prozent dieser Generation unterstützt wird, nicht dieselbe Rolle zukommt wie der SPD für die Nahkriegsgeneration, der fast die Hälfte der Wahlberechtigten dieser Generation zuneigten. Die Generation Y sollte etwa zwischen 1999 und 2013 in das Elektorat eintreten; signifikante Änderungen im Wahlverhalten zeigen sich aber erst bei der Wahl 2009. Zwei Hypothesen sind möglich: (1) Diese Generation ist zahlenmäßig deutlich geringer als die Vorgängergenerationen, weshalb sich Änderungen erst bemerkbar machen, wenn diese

den Bundeswahlleiter eine Stichprobe von etwa 2.700 Wahlbezirken ausgewählt, die mindestens 300 Wahlberechtigte umfassen. Im Jahr 1994 und 1998 wurde diese aus datenschutzrechtlichen Bedenken ausgesetzt.

32 Vgl. Rolf Becker, Walbeteiligung im Lebensverlauf. A-P-K-Analysen für die Bundesrepublik Deutschland in der Zeit von 1953-1987, in: Kölner Zeitschrift für Soziologie und Sozialpsychologie, 54. Jg. (2001), H. 2, S. 246 - 263, S. 248 f.

33 Bis zur Bundestagswahl 1972 lag das Wahlalter bei 20 Jahren und wurde erst bei dieser Wahl auf 18 Jahre gesenkt. 


\begin{tabular}{|c|c|c|c|c|c|c|c|c|c|c|c|c|c|c|}
\hline \multirow{2}{*}{$\begin{array}{l}\text { Tabelle 2: } \\
\text { Partei }\end{array}$} & \multicolumn{14}{|c|}{$\begin{array}{l}\text { Ergebnisse der Jungwähler in der repräsentativen Wablstatistik } 1953 \text { bis } 2009 \\
\text { (altes Bundesgebiet und Berlin West*, in Prozent) }\end{array}$} \\
\hline & & 1957 & 1961 & 1965 & \begin{tabular}{|l|}
1969 \\
\end{tabular} & 1972 & \begin{tabular}{|l|}
1976 \\
\end{tabular} & 1980 & \begin{tabular}{|l|}
1983 \\
\end{tabular} & 1987 & 1990 & 2002 & 2005 & 20 \\
\hline & & & & & & & & & & & & & & \\
\hline & 45,0 & 49,5 & 46,2 & 49,4 & 43,1 & 35,3 & 40,2 & 34,4 & 41,2 & 36,0 & 36,1 & 34,3 & 28,5 & 26,4 \\
\hline & & 7,3 & 2,0 & & 6,5 & & 8,5 & 11,4 & & & 10,2 & 10,0 & 11,2 & 15 \\
\hline & - & - & - & - & - & - & - & - & - & - & - & 1,6 & 4,6 & \\
\hline & - & - & - & - & - & - & - & 4,8 & 13,9 & 15,5 & 9,8 & 12,2 & 11,9 & 16,5 \\
\hline Sonst & 13,7 & 8,6 & 3,8 & 2,7 & 4,0 & 1,0 & 1,4 & 0,6 & 0,6 & & 6,8 & & 5,9 & 14,4 \\
\hline \multicolumn{15}{|c|}{$\begin{array}{l}\text { Anmerkung: Das Wahlalter betrug bis zur Wahl } 197221 \text { Jahre. Seit } 1972 \text { wird die Stimmabgabe der } \\
\text { Altersgruppen ab } 25 \text { Jahre im 10-Jahresintervall erfasst. Im Jahr } 1994 \text { und } 1998 \text { wurde keine Statistik } \\
\text { erhoben. } \\
\text { * Bis 1990: Nur altes Bundesgebiet; anschließend: altes Bundesgebiet und Berlin West. } \\
\text { Quelle: Bundeswahlleiter. }\end{array}$} \\
\hline
\end{tabular}

Generation vollständig wahlberechtigt ist. ${ }^{34}$ (2) Die Ergebnisse von 2009 sind zufällig und stellen keinen Trend dar.

Die Wahlbeteiligung erreichte in den 1970er Jahren ihren Höchststand mit über 90 Prozent und fiel seither stetig ab (vgl. Tabelle 3). Im Hinblick auf die Generationengruppen lassen sich folgende Tendenzen nachweisen:

- Bis zu den 1970er Jahren liegt die Wahlbeteiligung unter den Jungwählern etwa bei 80 Prozent.

- Ab 1972 steigt sie unter den Jungwählern weiter an und bleibt bis 1983 relativ hoch, bei über oder knapp unter 80 Prozent.

- Ab der Wahl 1987 nimmt die Wahlbeteiligung stark ab auf etwa 70 Prozent.

- Bei der Wahl 2009 zeigt sich ein dramatischer Rückgang der Wahlbeteiligung auf nurmehr knapp über 60 Prozent.

Für die Nachkriegs- und Wohlstandsgeneration lassen sich somit nicht nur enge Bindungen an die großen Parteien nachweisen, sondern auch eine starke Neigung, an Wahlen zu partizipieren. Die Generation X hingegen zeigt nicht nur nachlassende Bindungen an diese Parteien, sondern auch eine deutlich geringere Bereitschaft, sich überhaupt an Wahlen zu beteiligen. Ab 2009 zeigt sich sowohl eine signifikante Änderung des Wahlverhaltens als auch der Wahlbeteiligungsrate, die mit rund 61 Prozent den niedrigsten Stand überhaupt aufweist. Allerdings muss auch hier unklar bleiben, ob bei der Generation Y generell eine noch schwächere Beteiligungsrate vorliegt als bei ihrer Vorgängergeneration, oder ob das Ergebnis für 2009 zufällig ist und keinen Trend darstellt.

Die genauere Betrachtung im Zeitverlauf scheint zu bestätigen, dass das für die einzelnen Generationen zu beobachtende Wahlverhalten bei Eintritt in das Elektorat über die Zeit beibehalten wurde. Da sich die Altersgruppen der repräsentativen Wahlstatistik nicht mit den gewählten Geburtenkohorten decken, lässt sich das Wahlverhalten der Generationen

34 So war bei der Wahl des Jahres 2002 erst die Hälfte dieser Generation, die 18- bis 21-Jährigen in der hier erfassten Altersgruppe der unter 25-Jährigen vertreten. Erst bei der Wahl 2009 waren in dieser Altersgruppe keine Angehörigen der Generation X mehr vertreten. 


\begin{tabular}{|c|c|c|c|c|c|c|c|c|c|c|c|c|c|c|}
\hline \multirow[t]{2}{*}{ Tabelle 3: $\quad \begin{array}{l}1 \\
b\end{array}$} & \multicolumn{14}{|c|}{$\begin{array}{l}\text { Wablbeteiligung nach Altersgruppen, Ergebnisse der repräsentativen Wablstatistik } 1953 \\
\text { bis } 2009 \text { (altes Bundesgebiet und Berlin West*, in Prozent) }\end{array}$} \\
\hline & 1953 & 1957 & 1961 & 1965 & 1969 & 1972 & 1976 & 1980 & 1983 & 1987 & 1990 & 2002 & 2005 & 2009 \\
\hline $\begin{array}{l}21 \text { bis } \\
30 \text { Jahre }\end{array}$ & 79,9 & 82,6 & 81,8 & 79,3 & 79,0 & & & & & & & & & \\
\hline $\begin{array}{l}18 \text { bis } \\
25 \text { Jahre }\end{array}$ & & & & & & 84,5 & 83,5 & 79,7 & 82,9 & 75,0 & 65,3 & 71,3 & 69,1 & 62,6 \\
\hline Gesamt & 86,3 & 87,8 & 87,4 & 85,9 & 86,1 & 90,8 & 90,4 & \begin{tabular}{|l|}
87,6 \\
\end{tabular} & 88,4 & 83,1 & 76,5 & 81,2 & 79,2 & 72,9 \\
\hline $\begin{array}{l}\text { Differenz zum } \\
\text { Durchschnitt }\end{array}$ & 6,4 & 5,2 & 5,6 & 6,7 & 7,1 & 6,3 & 6,9 & 7,9 & 5,5 & 8,2 & 11,2 & 9,9 & 10,1 & 10,3 \\
\hline
\end{tabular}

nicht durchgängig beobachten und kann folglich nur unzureichend Aufschluss über generationelles Wahlverhalten erlangt werden.

Auch im Falle der Wahlbeteiligung lässt sich eine langfristige Beibehaltung der Partizipationsmuster erkennen, wenngleich auch hier eindeutige Schlüsse angesichts der Datenlage nicht möglich sind. Auffällig ist aber, dass bei allen Wahlen die Beteiligung der Altersgruppe $70+$ um etwa zehn Prozent unter jener der Altersgruppe 65 bis 70 Jahre liegt. Hierbei handelt es sich um einen Lebenszykluseffekt in dem Sinne, dass ältere Wähler aufgrund von Erkrankungen, Gebrechlichkeit etc. offensichtlich nicht mehr in gleicher Weise an Wahlen partizipieren können, wie jüngere Altersgruppen dies tun. 35

\section{Umfrageergebnisse und Wahlentscheid der Generationengruppen}

Die Analyse der repräsentativen Wahlstatistik zeigt, dass die einzelnen Generationen bei Eintritt in das Elektorat ein je spezifisches Wahlverhalten aufweisen. Die folgende Analyse soll klären, ob es sich hierbei lediglich um das Resultat eines bestimmten Lebensabschnitts handelt oder um das einer gemeinsamen Generationenzugehörigkeit. 36

\subsection{Methodik}

Der nachfolgenden Analyse liegen Daten der Allgemeinen Bevölkerungsumfrage der Sozialwissenschaften (ALLBUS) aus den Jahren 1980 bis 2012 zugrunde. Durch diese Umfrage werden in der Regel alle zwei Jahre Daten über Einstellungen, Verhaltensweisen und Sozialstruktur der Bevölkerung in der Bundesrepublik Deutschland erfasst, wobei größtmöglicher Wert auf eine repräsentative und ausreichend große Stichprobe gelegt wird.

Primär wird auf die kumulierten Ergebnisse der Umfragen von 1980 bis 2010 zurückgegriffen. Durch die Verwendung von zusammengefassten Daten unterschiedlicher Befragun-

$35 \mathrm{Zu}$ diesem Effekt vgl. ausführlich Laura Konzelmann / Corina Wagner / Hans Rattinger, Turnout in Germany in the Course of Time: Life Cycle and Cohort Effects on Electoral Turnout from 1953 to 2049, in: Electoral Studies, 31. Jg. (2012), H. 2, S. 250 - 261.

36 Vgl. hierzu auch Jürgen W. Falter, a.a.O. (Fn. 3), S. 3 f. 
gen aus verschiedenen Jahren wird sichergestellt, dass kurzfristige Trends im Wahlverhalten, etwa aufgrund spezifischer Ereignisse oder tagespolitischer Themen und Debatten, keinen überproportional großen Einfluss auf die Ergebnisse ausüben. Allerdings muss hierbei beachtet werden, dass die Geburtsjahrgänge bis 1962 in allen für den kumulierten Datensatz verwendeten Umfragen vertreten sind, während die späteren Geburtsjahrgänge erst ab dem jeweiligen Erreichen der Volljährigkeit Berücksichtigung finden. Um aussagekräftige Ergebnisse für die späteren Geburtsjahrgänge der Generationen X und Y zu erhalten, die naturgemäß in den ab 1980 erhobenen Daten unterrepräsentiert sind, wurden Daten der aktuellen Umfrage von 2012 ergänzend hinzugezogen.

Herausgefiltert wurden anhand des Geburtsjahres die hier zu untersuchenden vier Generationen, die auf ihr Wahlverhalten und spezifische Merkmale hin betrachtet wurden. Hierbei wurden ausschließlich in den alten Bundesländern erhobene Daten berücksichtigt, um die Vergleichbarkeit der vor und nach der Wiedervereinigung erhobenen Daten sicherzustellen und um Verzerrungen aufgrund der deutlichen Unterschiede im Wahlverhalten zwischen den alten und den neuen Bundesländern zu vermeiden. Für die betrachteten Altersgruppen wurde zudem die Repräsentativität in Bezug auf ihren Anteil an der bundesdeutschen Bevölkerung sowie die Alters- und Geschlechterverteilung geprüft.

\subsection{Die Generationengruppen in der allgemeinen Bevölkerungsumfrage}

(1) Nachkriegsgeneration: Geburtsjahr 1934 bis 1949

Die zwischen 1934 und 1949 geborenen Personen stellen 34,8 Prozent der Stichprobe aus den kumulierten Daten. Zum Zeitpunkt der Befragungen waren diese Teilnehmer zwischen 31 und 76 Jahre alt.

Ein Blick auf das formale Bildungsniveau dieser Altersgruppe zeigt, dass 57,2 Prozent einen Volks- oder Hauptschulabschluss besaßen. Über ein abgeschlossenes Studium verfügten dagegen nur 9,9 Prozent dieser Altersgruppe.

15,1 Prozent gaben an, Mitglied einer Gewerkschaft zu sein. In der Vergangenheit war allerdings fast die Hälfte der befragten Personen (44,6 Prozent der gültigen Antworten) gewerkschaftlich organisiert gewesen. 4,9 Prozent gaben an, einer politischen Partei anzugehören.

Als im Vergleich auffallend hoch zeigt sich die Bereitschaft dieser Generation, wählen zu gehen. So hatten 91,5 Prozent bei der jeweils letzten Bundestagswahl vor der Befragung ihre Stimme abgegeben. Bei der jeweils letzten Landtagswahl lag die Wahlbeteiligung in dieser Gruppe sogar bei 93,7 Prozent. Hierbei erklärten 66,6 Prozent, eine klare Parteipräferenz zu besitzen, wobei vor allem die beiden Volksparteien angegeben wurden: 45,7 Prozent präferierten die Unionsparteien, 41,1 Prozent die SPD. Mit der FDP sympathisierten nach eigener Angabe sechs Prozent dieser Generation. Im Vergleich zu den anderen Wählergenerationen ist der Anteil derjenigen, die Bündnis 90/Die Grünen präferierten, mit 2,5 Prozent sehr niedrig.

Wird dagegen nach der konkreten Wahlabsicht für die jeweils nächste Bundestagswahl gefragt, so würden die Grünen von 6,8 Prozent gewählt werden, die FDP von 9,5 Prozent, die SPD von 35,4 und die CDU/CSU von 33,9 Prozent. 8,3 Prozent dieser Altersgruppe gaben an, bei der nächsten Bundestagswahl nicht wählen zu gehen. Bei der letzten Befragung 
von 2012, die konkret nach der Wahlabsicht für die nächste Bundestagswahl im September 2013 fragte, würden die Unionsparteien in dieser Altersgruppe mit 38,7 wiederum deutlich vor der SPD mit 32,2 Prozent liegen.

(2) Wohlstandsgeneration: Geburtsjahr 1950 bis 1964

Die Wohlstandsgeneration der Zeit des Wirtschaftswunders macht 37,9 Prozent der Stichprobe aus und stellt damit die größte Gruppe, was aufgrund der hohen Geburtenraten zu Beginn der 1960er Jahre auch ihrem gesellschaftlichen Anteil in der Bundesrepublik entspricht. Zum Zeitpunkt der zugrunde liegenden Befragungen waren Teilnehmer aus dieser Altersgruppe zwischen 18 und 60 Jahre alt. Das Durchschnittsalter lag bei 37,4 Jahren.

Die formale Bildung liegt in dieser Gruppe bereits etwas höher als bei der zuvor betrachteten „Nachkriegsgeneration“. Der vorherrschende Schulabschluss ist hier die Mittlere Reife, über die 37,3 Prozent verfügten. Den von der vorhergehenden Generation noch am häufigsten genannten Volks- oder Hauptschulabschluss gaben 33,2 Prozent der Befragten aus der Wohlstandsgeneration als ihren höchsten formalen Bildungsabschluss an. Ein Hochschulstudium hatten 12,2 Prozent dieser Altersgruppe abgeschlossen.

Bezüglich des Organisationsgrades lässt sich für die Wohlstandsgeneration feststellen, dass 19,3 Prozent zum Zeitpunkt der Befragung Mitglied in einer Gewerkschaft und immerhin 38,3 Prozent in der Vergangenheit gewerkschaftlich organisiert waren. Mitglied einer Partei waren zum Befragungszeitpunkt 3,2 Prozent dieser Altersgruppe.

Bei der jeweils letzten Bundestagswahl vor der Befragung hatten nach eigenen Angaben 86,8 Prozent ihre Stimme abgegeben. Bei der jeweils letzten Landtagswahl vor der Umfrage lag die Wahlbeteiligung in dieser Gruppe mit 89,7 Prozent ebenfalls sehr hoch.

Eine klare Parteipräferenz zu besitzen hatten 59,2 Prozent der befragten Personen aus der Wohlstandsgeneration bekundet. Im Gegensatz zur Nachkriegsgeneration liegt diese allerdings deutlich stärker bei der SPD, die von 44,1 Prozent präferiert wurde. Die Unionsparteien wurden von 35 Prozent benannt. Mit 3,2 Prozent lagen auch hier die Werte für die Liberalen unterhalb derjenigen der vorherigen Jahrgänge. Die Grünen wurden dagegen von 11,4 Prozent als Präferenz angegeben.

Auf die Frage nach der konkreten Wahlabsicht für die nächste Bundestagswahl nach der jeweiligen Befragung äußerten 33,7 Prozent, die SPD wählen zu wollen. Die Unionsparteien kämen in dieser Altersgruppe auf durchschnittlich 24,3 Prozent, die Grünen würden von 15,5, die FDP von acht Prozent gewählt werden. Entsprechend der erkennbaren Tendenz zu linken Parteien käme die PDS (ab 2007 Die Linke) auf 5,1 Prozent. 10,9 Prozent dieser Altersgruppe gaben an, bei der nächsten Bundestagswahl nicht wählen gehen zu wollen. Bei der Befragung 2012 lagen SPD und CDU etwa gleichauf mit 26,2 Prozent beziehungsweise 27,7 Prozent. Die Grünen kämen hier auf 18,2 Prozent.

(3) Generation X: Geburtsjahr 1965 bis 1980

Die zwischen 1965 und 1980 geborenen Mitglieder der „Generation X“ machten mit 8.824 befragten Personen 21 Prozent der Stichprobe aus. Ihr Durchschnittsalter lag bei 28,5 Jahren, wobei die befragten Personen zum Zeitpunkt der Befragung zwischen 18 und 45 Jahre alt waren. 
Das formale Bildungsniveau liegt hier wiederum über dem der zuvor betrachteten Generationen: 41 Prozent gaben die Mittlere Reife als höchsten Bildungsabschluss an. Die Hochschulreife war mit 25,4 Prozent der danach am häufigsten genannte Schulabschluss. Einen Hochschulabschluss wiesen 9,2 Prozent der Befragten aus der Generation X auf. Dieser Wert erscheint zunächst gering, doch ist hier das niedrige Alter dieser Gruppe zum Zeitpunkt der Datenerhebungen zu berücksichtigen. So konnte diese Altersgruppe bei den Befragungen von 1980 beispielsweise noch nicht berücksichtigt werden, und auch bei den späteren Befragungen war ein Großteil noch in der Ausbildung. So gaben 39,2 Prozent an, sich zum Zeitpunkt der Befragung in einer Berufsausbildung zu befinden, und 12,1 Prozent studierten an einer Universität oder Fachhochschule.

Daher empfiehlt es sich, an dieser Stelle die Ergebnisse der aktuellen Befragung von 2012 hinzuzuziehen: Dabei lag der Anteil der Hochschulabsolventen innerhalb der Generation X bei 19,1 Prozent. Ganze 33 Prozent gaben an, die allgemeine Hochschulreife zu besitzen, 9,4 Prozent die Fachhochschulreife, und der Anteil derjenigen, die mit der Mittleren Reife ihre Bildung abgeschlossen hatten, lag mit 33,5 Prozent nur noch leicht über den Angaben zur allgemeinen Hochschulreife.

Mitglied einer Gewerkschaft waren zum Zeitpunkt der Befragung 12,4 Prozent, und lediglich 17 Prozent gaben an, zuvor einmal einer Gewerkschaft angehört zu haben. Sich in einer politischen Partei zu engagieren, bekundeten dagegen lediglich 2,2 Prozent der „Generation $X^{\prime \prime}$.

Eine feste Parteipräferenz besaßen dabei laut eigener Angabe nur 50,7 Prozent dieser Generation. Am häufigsten wurden hier die Unionsparteien genannt (37,2 Prozent), dicht gefolgt von der SPD (36,5 Prozent). 12,6 Prozent erklärten, eine Präferenz für die Grünen zu besitzen, 6,4 Prozent für die PDS/Die Linke und 5,3 Prozent für die FDP. Wurde nach der konkreten Wahlabsicht für die nächste Bundestagswahl gefragt, so lag die SPD mit 28,2 Prozent vor der CDU/CSU mit 24,1 Prozent. Die Grünen würden von 16,3 Prozent, die FDP von acht Prozent gewählt werden, die PDS/Linkspartei käme auf 5,3 Prozent. 13,8 Prozent dieser Altersgruppe wollten nicht wählen gehen.

77,4 Prozent gaben an, bei der jeweils letzten Bundestagswahl vor der Befragung ihre Stimme abgegeben zu haben, und 80,1 Prozent taten dies bei der der Befragung vorhergehenden Landtagswahl. Bei der letzten Befragung 2012 fällt vor allem auf, dass die Grünen mit 22,5 Prozent vor der SPD mit 20,6 Prozent lagen.

\section{(4) Generation Y: Geburtsjahr 1981 bis 1995}

Die Mitglieder dieser Altersgruppe wurden erst während des Untersuchungszeitraumes geboren und finden daher erst bei den späteren Befragungen Eingang in den Datensatz und das Elektorat. Hinzu kommt, dass der Datensatz nur Erhebungen bis 2010 enthält, bei denen ausschließlich volljährige Probanden befragt wurden. Deshalb finden die Geburtsjahrgänge nach 1991 ebenfalls keine Berücksichtigung. Entsprechend niedrig ist der Anteil der „Generation Y“ an der Stichprobe mit 1.680 befragten Personen (vier Prozent). Die berücksichtigten Teilnehmer waren zum Zeitpunkt der Datenerhebungen zwischen 18 und 29 Jahre alt, das Durchschnittsalter der Generation Y betrug in der vorliegenden Stichprobe 21,6 Jahre. Der am häufigsten angegebene Schulabschluss war auch hier die Mittlere Reife (33,3 Prozent) gefolgt von der Hochschulreife (31,0 Prozent). Der Anteil der Hochschulabsolventen lag bei 4,5 Prozent, wobei auch hier von einem größeren Anteil an Teilnehmern 
ausgegangen werden muss, die sich zum Zeitpunkt der Befragung noch in einem Studium befanden. 37

Angesichts der relativ geringen Anzahl von Befragten der Generation Y im vorliegenden kumulierten Datensatz empfiehlt es sich wiederum, die aktuellen Daten der Befragung von 2012 hinzuzuziehen. Hier wird ein offensichtlicher Anstieg des Anteils der Befragten mit Hochschulreife deutlich: 36,9 Prozent machten diese Angaben. Die Mittlere Reife stellte mit 30,1 Prozent den nächst häufig genannten Schulabschluss dar. Einen Hochschulabschluss zu besitzen, wurde 2012 von 13,0 Prozent der Befragten dieser Altersgruppe angegeben. Fünf Prozent waren noch Schüler. Die Jahrgänge 1994 und 1995 wurden nicht berücksichtigt, so dass auch hier von einem formalen Bildungsniveau ausgegangen werden kann, das nochmals über dem der vorhergehenden Generation liegt.

Der Organisationsgrad der Generation Y rangiert unterhalb der Werte der vorhergehenden Generationen, wobei auch hier das niedrige Durchschnittsalter einen wesentlichen Faktor darstellen dürfte: 6,1 Prozent gaben an, zum Zeitpunkt der Befragung Mitglied in einer Gewerkschaft zu sein, lediglich zwei Prozent waren dies zuvor einmal gewesen. Einer Partei gehörten lediglich 1,5 Prozent der Befragten dieser Altersgruppe an. In den Ergebnissen der Befragung von 2012 lag dieser Wert mit 2,7 Prozent auf dem Niveau der vorhergehenden Generation X.

Gemäß den Angaben aus den kumulierten Daten lag die Beteiligung an Bundestagswahlen in dieser Generation bei durchschnittlich 64,8 Prozent. Eine eindeutige Parteipräferenz besaßen nach eigener Angebe 42,7 Prozent der befragten Personen. Meistgenannte Partei war hierbei die SPD mit 40,6 Prozent; die Unionsparteien wurden von 32,2 Prozent als Präferenz angegeben. Die Grünen kamen auf 11,3 Prozent, die PDS/Linkspartei auf 7,1 und die FDP auf 5,9 Prozent.

Wird nach der konkreten Wahlabsicht für die nächste Bundestagswahl gefragt, so lagen die Unionsparteien mit 26,8 Prozent knapp vor der SPD mit 25,9 Prozent. Die Grünen wurden von 15,3 Prozent, die FDP von 8,7 und die PDS/Linkspartei von 6,7 Prozent präferiert. 12,7 Prozent würden nach eigener Angabe nicht wählen gehen.

Die 2012 erhobenen Daten weichen hier deutlich ab. Allerdings handelt es sich dabei um die konkrete Frage nach der Wahlabsicht für die kommende Bundestagswahl im September 2013 und nicht um zusammengefasste Daten aus Befragungen, die in verschiedenen Jahren durchgeführt wurden. Insofern sind diese Angaben stärker dem Einfluss tagespolitischer Trends und Ereignisse unterworfen, die kurzfristig die Sympathiewerte einzelner Parteien enorm beeinflussen können. Die SPD war hier die am häufigsten genannte Partei: 27,1 Prozent der Befragten gaben an, die Partei wählen zu wollen, gefolgt von den Unionsparteien mit 21,9 Prozent. Die Grünen stellen in dieser Altersgruppe mit 14,2 Prozent die drittstärkste Partei dar, dicht gefolgt von der NPD mit 13,4 Prozent. Die Linke wurde dagegen nur von 4,1 Prozent dieser Altersgruppe als Präferenz benannt. 10,7 Prozent gaben 2012 an, bei der nächsten Bundestagswahl voraussichtlich nicht wählen zu gehen.

Für die Bundestagswahl am 22. September 2013 lag noch keine vergleichbare Datenbasis vor, so dass diese Ergebnisse noch nicht umfassend einbezogen werden konnten. Doch trotz wieder leicht gestiegener Wahlbeteiligung und eines Rekordergebnisses der Unionsparteien zeigen sich auch hier deutlich die dargelegten Tendenzen im Wahlverhalten: Stütze des

37 Wurde in den kumulierten ALLBUS-Daten 1980 bis 2010 für diese Geburtsjahrgänge nicht erfasst. 


\begin{tabular}{|c|c|c|c|c|}
\hline Generation & $\begin{array}{l}\text { Nachkriegs- } \\
\text { generation }\end{array}$ & $\begin{array}{l}\text { Wohlstands- } \\
\text { generation }\end{array}$ & Generation X & Generation Y \\
\hline Jahrgänge & 1934 bis 1949 & 1950 bis 1964 & 1965 bis 1980 & 1981 bis $1995^{* *}$ \\
\hline $\begin{array}{l}\text { Gültiger Anteil an Stichprobe } \\
1980 \text { bis } 2010(\mathrm{~N}=54.243)\end{array}$ & 35,6 & 37,9 & 21,0 & 4,0 \\
\hline $\begin{array}{l}\text { Gültiger Anteil an Stichprobe } \\
2012(\mathrm{~N}=3.480)\end{array}$ & 17,7 & 27,5 & 26,1 & 20,2 \\
\hline \multicolumn{5}{|l|}{ Bildung } \\
\hline $\begin{array}{l}\text { Meistgenannter Schul- } \\
\text { abschluss } \\
\text { Anteil an Altersgruppe } \\
\text { Akademikeranteil }\end{array}$ & $\begin{array}{c}\text { Volks- / } \\
\text { Hauptschule } \\
57,2 \\
9,9 \\
\end{array}$ & $\begin{array}{l}\text { Mittlere } \\
\text { Reife } \\
37,3 \\
12,2 \\
\end{array}$ & $\begin{array}{c}\text { Mittlere } \\
\text { Reife } \\
41,0 \\
19,1 \\
\end{array}$ & $\begin{array}{l}\text { Hochschul- } \\
\text { reife } \\
36,9 \\
13,0^{*}\end{array}$ \\
\hline \multicolumn{5}{|l|}{ Organisationsgrad } \\
\hline Mitglied in Gewerkschaft & 15,1 & 19,3 & 12,4 & 6,1 \\
\hline Früher in Gewerkschaft aktiv & 44,6 & 38,3 & 17,0 & 2,0 \\
\hline Mitglied in Partei & 4,9 & 3,2 & 2,2 & 1,5 \\
\hline Wahlbeteiligung BTW & 91,5 & 86,8 & 77,4 & 64,8 \\
\hline Feste Parteipräferenz & 66,6 & 59,2 & 50,7 & 42,7 \\
\hline \multicolumn{5}{|l|}{ Parteipräferenz } \\
\hline CDU/CSU & 45,7 & 35,0 & 37,2 & 32,2 \\
\hline SPD & 41,1 & 44,1 & 36,5 & 40,6 \\
\hline Grüne & 2,5 & 11,4 & 12,6 & 11,3 \\
\hline FDP & 6,0 & 3,2 & 5,3 & 5,9 \\
\hline PDS/Die Linke & 3,8 & 4,4 & 6,4 & 7,1 \\
\hline Sonstige & 0,9 & 1,9 & 2,0 & 2,9 \\
\hline Nichtwähler & 8,3 & 10,9 & 13,8 & $12,7^{* *}$ \\
\hline \multicolumn{5}{|c|}{$\begin{array}{l}\text { * Viele Teilnehmer befanden sich zum Zeitpunkt der Befragung noch in einer Ausbildung oder einem } \\
\text { Studium. } \\
\text { ** Die Jahrgänge nach 1991/1993 waren im Untersuchungszeitraum noch nicht volljährig und bleiben } \\
\text { daher unberücksichtigt. } \\
\text { Quelle: Eigene Berechnung. }\end{array}$} \\
\hline
\end{tabular}

Unionssieges waren Wähler über 60. Innerhalb dieser Gruppe erhielt die CDU/CSU knapp die Hälfte der Stimmen (49 Prozent). Gleichzeitig erreichte der Anteil der Parteien, die an der Fünf-Prozent-Hürde scheiterten und somit nicht in den Bundestag einziehen, mit insgesamt 15,7 Prozent ein Rekordniveau. Auch wenn man den knapp verpassten Einzug der bisherigen Regierungspartei FDP, der eine historische Zäsur darstellt, hier nicht hinzuzählt, liegt der Anteil der „Sonstigen“ mit 10,9 Prozent der gültigen Zweitstimmen noch immer sehr hoch, was vor allem dem Erfolg der erst im April 2013 gegründete Alternative für Deutschland (AfD) zuzuschreiben ist, die mit 4,7 Prozent den Einzug in den Bundestag nur knapp verpasste. Aus der Gruppe der 18- bis 29-Jährigen gaben sogar 20 Prozent ihre Stimme einer der Kleinstparteien. 38

38 Vgl. Forschungsgruppe Wahlen, Wahlanalyse Bundestagswahl 2013, Mannheim 2013, http:// www.forschungsgruppe.de/Aktuelles/Bundestagswahl/News_BTW_2013.pdf (Abruf am 27. November 2013). 


\section{Fazit: Der generationelle Wandel als Herausforderung der etablierten Parteien}

Die anhand der repräsentativen Wahlstatistik und der Daten der allgemeinen Bevölkerungsumfrage der Sozialwissenschaften betrachteten Wählergenerationen weisen ein deutlich unterschiedliches Wahlverhalten auf und bestätigen die aufgezeigten Trends des Wählerverhaltens sowie die eingangs formulierten Thesen.

In der Nachkriegsgeneration zeigte sich eine klare Präferenz für die beiden Volksparteien. Zudem erhielt die FDP in dieser Altersgruppe den stärksten Zuspruch und war die am dritthäufigsten genannte Partei. Dies entspricht dem deutschen Parteiensystem zur Zeit der Sozialisationsphase dieser Generation. Generell wurden die Unionsparteien etwas häufiger präferiert als die SPD, was sich ebenfalls mit der eingangs formulierten These zur Wahl der Parteien mit zugesprochener Wirtschaftskompetenz deckt. Die Bereitschaft zur politischen Partizipation ist in dieser Generation stark ausgeprägt. Sie stellte in den verwendeten Umfragedaten die meisten Parteimitglieder, wies eine hohe gewerkschaftliche Organisiertenrate auf sowie die höchste Wahlbeteiligung.

Die von wirtschaftlichem Aufstieg und sozialer Sicherheit geprägte Wohlstandsgeneration stand der SPD sehr nahe, was dem Wunsch nach ökonomischer Teilhabe entspricht. Auch diese Generation zeigte eine sehr hohe Bereitschaft zur Wahl. Ebenso wie in der Vorgängergeneration war ihr gewerkschaftlicher Organisierungsgrad sehr hoch.

Auch die eingangs aufgestellten Thesen zum politischen Verhalten der Generation X, deren gewerkschaftlicher und politischer Organisationsgrad deutlich unter den Werten der Vorgängergenerationen lag, konnten bestätigt werden. So stellte sie den höchsten Anteil potentieller Nichtwähler in der Stichprobe, und auch bezüglich der Parteipräferenz taten sich deutliche Unterschiede zur vorhergehenden Wohlstandsgeneration auf: Es zeigten sich erheblich schwächere Bindungen an die beiden großen Volksparteien, wobei SPD und CDU/CSU fast gleichauf lagen. Gleichzeitig wies die Generation X aber eine starke Affinität zu den Grünen auf, die angesichts der prognostizierten postmaterialistischen Werteeinstellung dieser Generation zu erwarten war. Nach der Wiedervereinigung lässt sich zudem eine deutliche Tendenz zur Wahl der Partei Die Linke erkennen, und auch die FDP schneidet besser ab als bei der Vorgängergeneration.

In der Generation Y fand sich der geringste Anteil an befragten Personen, die sich in einer Partei oder Gewerkschaft engagierten. Allerdings ist zu berücksichtigen, dass sich etliche in dieser Altersgruppe zum Zeitpunkt der letzten Befragung noch in der Ausbildungsphase befanden und die jüngsten Jahrgänge nicht berücksichtigt wurden. Die bis 2010 erfassten Parteipräferenzen zeigten eine Affinität zu den Parteien, die traditionell zum linken ideologischen Spektrum gezählt werden (SPD, Grüne, Die Linke). Diese scheinen sich allerdings nicht ohne weiteres auf die konkrete Wahlabsicht übertragen zu lassen. So gaben 2012 fast fünfzehn Prozent dieser Altersgruppe an, ihre Stimme bei der nächsten Bundestagswahl der rechtsextremen NPD geben zu wollen. Generell lag hier der Anteil derjenigen, die bereit waren, für eine der „sonstigen“ Parteien zu votieren, deutlich über dem Niveau aller vorhergehenden Generationen. Ob es sich hierbei um eine kurzfristige Absicht zur Protestwahl handelt, oder ob bei dieser Generation generell eine ausgeprägte Ablehnung der etablierten Parteien und konventioneller Formen der politischen Partizipation besteht, konnte angesichts der Datenlage nicht abschließend festgestellt werden. Da noch nicht alle zu dieser Generation gezählten Jahrgänge im Elektorat vertreten sind, konnte auch der Anteil der potentiellen Nichtwähler nicht abschließend ermittelt werden; jedoch lag er bereits jetzt 
knapp unter dem Niveau der vorhergehenden Generation X, und auch die repräsentative Wahlstatistik deutet auf eine nur schwach ausgeprägte Tendenz zur politischen Partizipation. Die These von der generationellen Wasserscheide, das heißt eine sich stetig steigernde Affinität zu den Grünen, scheint sich indes nicht zu bestätigen. Die Präferenz für die Grünen lag in dieser Generation sogar leicht unter jener der Generation X. Diese Generation weist - sollten sich die Tendenzen bestätigen - zwar somit ebenfalls eine spezifische Form der politischen Partizipation im Sinne von nur losen Parteipräferenzen und einer geringen Neigung zur Beteiligung an Wahlen auf; im Sinne Mannheims ist sie aber angesichts der unklaren politischen Präferenzen eher eine „politische Generation an sich“, nicht aber „für sich".

Hat man diese Tendenzen vor Augen, werden die Probleme, mit denen sich die beiden großen Parteien angesichts des demographischen Wandels konfrontiert sehen, schnell klar. Wie ausgeführt stellten Nachkriegs- und Wohlstandsgeneration im Jahr 2000 noch fast drei Viertel der Wahlberechtigten; 2010 waren es noch gut die Hälfte und im Jahr 2020 werden diese Generationen in der Minderheit sein. Lange konnten die beiden großen Parteien angesichts der großen Zahl an potentiellen Wählern uneingeschränkt auf die Nachkriegs- und die Wohlstandsgeneration setzen. Dies dürfte aber in Zukunft angesichts des demographischen Trends im Elektorat, der sich kurzfristig als Wechsel von den materialistisch geprägten Generationen des wirtschaftlichen Aufstiegs und gesellschaftlichen Wohlstands hin zu den geburtenschwachen, von sich verschlechternden Arbeitsmarktbedingungen und den Prozessen der Globalisierung geprägten Folgegenerationen bemerkbar macht, immer schwieriger werden. Da die Generationen X und Y, die deutlich schwächere Bindungen an die beiden Parteien besitzen und auch in einem erheblich geringeren Ausmaß gewerkschaftlich oder politisch organisiert sind, bald die Mehrheit des Elektorates stellen, ist zu erwarten, dass SPD und CDU sich bald als 20 Prozent-Parteien wiederfinden. Die etablierten Parteien müssen daher umsteuern, wollen sie ihre gesellschaftliche Verankerung nicht verlieren. Geboten wäre eine deutliche Verjüngung der Mitglieder - und vor allem des politischen Personals -, um Jüngeren Identifikation zu bieten und sie wieder für sich zu gewinnen. Zudem müsste es den etablierten Parteien stärker gelingen, solche Themen zu besetzen, anhand derer sie die junge Generation wieder an sich binden können.

Die These, dass die Grünen, die in der Vergangenheit ihre Wähler vor allem unter den Jüngeren fanden, mit dem generationellen Wandel im Elektorat die Rolle einer neuen Volkspartei übernehmen könnten, scheint sich indes nicht zu bestätigen, da die jüngsten Wahlberechtigten trotz ihrer hohen Bildung keine ausgesprochen Neigung zu ihnen zeigen. Die Daten lassen somit insgesamt den Schluss zu, dass Wahlausgänge immer unsicherer werden und Mehrheiten immer schwerer zu beschaffen sein dürften. Mindestens ist eine fortschreitende Instabilität des Parteiensystems zu erwarten, denn die Bereitschaft, sich neuen Parteien zuzuwenden oder sich ganz abzuwenden, wird unter den Wahlberechtigten immer größer. 